KIAS-P99044

June 28, 2018

\title{
Effective Field Theory For Nuclei, Dense Matter And The Cheshire Cat
}

\author{
Mannque Rho \\ School of Physics, Korea Institute for Advanced Study, Seoul 130-012, Korea \\ and \\ Service de Physique Théorique, CE Saclay, 91191 Gif-sur-Yvette, Francel \\ E-mail: rho@spht.saclay.cea.fr
}

\begin{abstract}
In this talk I discuss three related topics based on some of the recent developments in hadron and nuclear physics: one, effective field theory approach to two-nucleon systems; two, an explanation of the flavor singlet axial charge in the proton (i.e., "proton spin problem") in terms of a Cheshire Cat phenomenon; and three, the quark-hadron duality in hadronic matter at high density and "qualitons" at high density ("superqualitons"). The principal common theme in these discussions will be the emergence of the generic feature of the Cheshire Cat Principle.
\end{abstract}

${ }^{*}$ Talk given at the 12th Nuclear Physics Summer School and Symposium and the 11th International Light-Cone Workshop "New Directions in QCD," 21-25 June 1999, Kyungju, Korea

${ }^{\dagger}$ Permanent address 


\section{Introduction}

I would like to discuss in this talk three topics which superficially would look unrelated but in essence can be connected by the general theme of Cheshire Cat. I will first look at two-nucleon systems in terms of macroscopic variables of QCD, namely hadrons; I will then go back to an elementary hadron, in particular the proton and examine its microscopic structure by "punching" a hole in the proton and then putting quarks and gluons of the appropriate quantum numbers inside and argue that there is a continuity in the descriptions in terms of hadronic variables and in terms of quark-gluon variables; finally the continuity of quarks and hadrons at high density will be described in terms of qualitons - quark solitons. Many of my collaborators have contributed to the development discussed in this talk. Among them are Gerry Brown, Bengt Friman, Deog Ki Hong, Kuniharu Kubodera, Kurt Langfeld, Hee-Jung Lee, Dong-Pil Min, Byung-Yoon Park, Tae-Sun Park, Vicente Vento and Ismail Zahed.

\section{Two-Nucleon Systems in Effective Field Theory}

Consider two nucleon interactions at very low energy. We are generically interested in the energy-momentum probe much less than the pion mass $m_{\pi} \sim 140 \mathrm{MeV}$. At this energy, according to Weinberg's "theorem," the content of QCD can be phrased in terms of the nucleons and pions. In fact if we are probing a scale much less than the pion mass, we can even ignore the pions and work with the nucleons only. The corresponding framework is an effective field theory (EFT). Much work has been done on this EFT for two-nucleon systems [1, 2, 3, 田, 旬] (see [6] for recent reviews). There are two classes of observables to look at. One is scattering process and the other is response function to external fields. The two are of course complementary in revealing the physics involved. Recent efforts have been put more on scattering than on response functions although more information has traditionally been gained from the latter in nuclear physics. Both need to be treated simultaneously which I will do here.

In the literature so far, there are broadly two approaches to EFT in nuclear physics. One is the original Weinberg approach [1] where a systematic power counting is made only to the "irreducible graphs," for which chiral perturbation theory (with pions figuring crucially) becomes applicable in organizing the expansion and the reducible graphs are summed to all orders with the irreducible graphs entering as vertices. This scheme used in Ref. [1, 2, 3, 5] - which in spirit is close to the original Wilsonian EFT but incurs possible errors in the power counting - involves a scale $\Lambda \gtrsim m_{\pi}$ as the counting is applied only to the irreducible terms. I will call this the $\Lambda$ counting. The other approach 偛 motivated to account more transparently for the large s-wave scattering lengths in two-nucleon scattering purports to do a systematic counting for the S-matrix as a whole which amounts to summing all graphs involving leading non-derivative four-Fermi contact interactions while treating all 
others, including pion exchanges, as perturbation. This approach renders a more systematic accounting of the powers of $Q / \Lambda$ where $Q=\sqrt{M B}, p$ as well as $m_{\pi}$ but at the expense of certain predictivity. This is referred to as $Q$-counting scheme.

There have been lots of hot debates as to whether one scheme is more powerful and or more consistent than the other, mainly in connection with the scattering [6]. While the situation is not completely settled, I believe that it is fair to say that the two are both consistent with the tenet of EFT and roughly equivalent in its power. Eschewing the debate which seems somewhat academic, I will simply focus on the $\Lambda$-counting approach in this talk. In fact, in the processes that I will consider, I would claim that the $\Lambda$ counting is more adaptable to - and more predictive in treating - nuclear physics problems than the $Q$ counting. One great advantage of the $\Lambda$ scheme is that it allows one to calculate precisely defined corrections to what can be obtained from so-called realistic potential models (PM in short) that have been developed by nuclear theorists since a long time, thus giving the realistic potential models $(\mathrm{PM})$ a first-principle justification. It allows us to study processes involving not just few-body but also many-body systems. For instance, it is possible to calculate the "hep" process in the Sun $p+{ }^{3} \mathrm{He} \rightarrow{ }^{4} \mathrm{He}+e^{+}+\nu_{e}$ (which is currently an exciting issue after the recent Surperkamiokande neutrino data) with an accuracy that can be controlled systematically. It has also been successfully applied to calculating axial charge transitions of heavy nuclei (see [7]) with the additional ingredient of BR scaling [8].

While there is no definitive evidence that the $\Lambda$ scheme is fully justified for n-body systems with $n \gg 2$, it definitely works for $n=2$ systems. In Ref. [5], it has been shown in a cutoff regularization (with a finite cutoff as required by EFT [9]) that the EFT results of the leading order terms in all two-body observables at low energy $E \ll m_{\pi}$ are precisely reproduced by the potential model results. This is the case not only for scattering amplitudes but also all electroweak response functions. What EFT brings in addition to what we get from the PM is a systematic procedure to compute corrections to the leading order results. For low-energy processes, this status of the PM can be understood by the fact that the tail of the wave functions is a physical quantity and the realistic potential models which are fit to experiments have the correct asymptotic properties in the wave function. This point has also been stressed and clarified by Phillips and Cohen in a recent important paper [10].

Considered to order $Q^{n}$ where $n$ is the order in the $\Lambda$ counting (which I will consider relative to the leading order term in the expansion of the irreducible graphs), the s-wave scattering amplitudes are accurately postdicted [5, 11] up to $p \leq m_{\pi}$ for $n=2$ and a cutoff appropriate to the number of pions exchanged (one or two) in the irreducible graphs. Deuteron properties are also well understood within the same scheme [5]. The scheme allowed the calculations to order $Q^{2}$ and $Q^{3}$ of the proton fusion process in the Sun [12]

$$
p+p \rightarrow d+e^{+}+\nu_{e}
$$

and of the threshold np capture [2, 13] with polarized projectile and target nucleons

$$
\vec{n}+\vec{p} \rightarrow d+\gamma
$$


The process (1) crucial for the solar neutrino problem is given in the scheme to an accuracy of $1 \sim 3$ percents (the uncertainty here is due to the exchange current that appears at order $Q^{3}$ ). The unpolarized cross section for (2) has been computed to the accuracy of 1 percent in a complete agreement with the experiment. More significantly, the polarization observables $P$ (circular polarization) and $\eta$ (anisotropy) have been predicted parameter-free in Ref. 13] This is a genuine prediction since there are no experimental data available. (They are currently being measured in ILL of Grenoble [15].)

In all these postdictions and predictions, there is very little $\Lambda$ dependence as required by the tenet of EFT. This is a clear indication that the scheme is fully consistent.

One can go up in the momentum range by doing higher order calculations. Phillips and Cohen [10] discuss how the two-body EM form factors can be described in the $\Lambda$ scheme. Pushing somewhat the validity of the scheme, one can calculate even the process

$$
e+d \rightarrow e+n+p
$$

involving large momentum transfers $q \gtrsim 1 \mathrm{GeV}$. In fact this process measured in 1980's at ALS of Saclay and elsewhere is considered to be the unambiguous confirmation of mesonexchange currents in nuclei (see [17]).F2

\section{3 "Proton Spin" and The Cheshire Cat}

The nucleons that figured in the above section were color-singlet point-like fields that say nothing explicit about quarks and gluons. Let me now imagine puncturing a hole of size of radius $R$ in the proton and populating the inside with the QCD degrees of freedom, quarks and gluons. How to do this consistently with QCD is known and the model involved is the chiral bag (this is described extensively in [18]) which consists of a quark-gluon sector inside the bag and a color-singlet hadronic sector outside, with the two sectors connected by suitable boundary conditions. When constructed with relevant degrees of freedom and in consistency with the symmetries of QCD, the model gives what is now called "Cheshire Cat." In short, the Cheshire Cat Principle [18] states that at low energy, physics involving hadrons should be independent of the bag size $R$. It has been shown that this principle is operative semi-quantitatively in all properties of the nucleon [19] except for the flavor-singlet axial charge (FSAC) of the proton which is related to the "proton spin." Here I will briefly describe - leaving the details to the paper by Lee et al [21] - how this Cheshire Cat property can be recovered in the FSAC when chiral symmetry and chiral anomaly are judiciously

\footnotetext{
${ }^{\# 1}$ A similar prediction in the $Q$ scheme was made by Chen, Rupak and Savage [14].

\#2 When I suggested this process at the second INT-Caltech EFT meeting as a case for testing the EFT strategy in the $Q$ counting, everyone (!) in the audience chuckled and said the process is completely out of reach for EFT (see 16]). I grant that this may be true in the $Q$ scheme at least for the moment but not in the $\Lambda$ scheme where it has worked stunningly, confirming the "chiral filter hypothesis." Voilà the power of the $\Lambda$ scheme!
} 


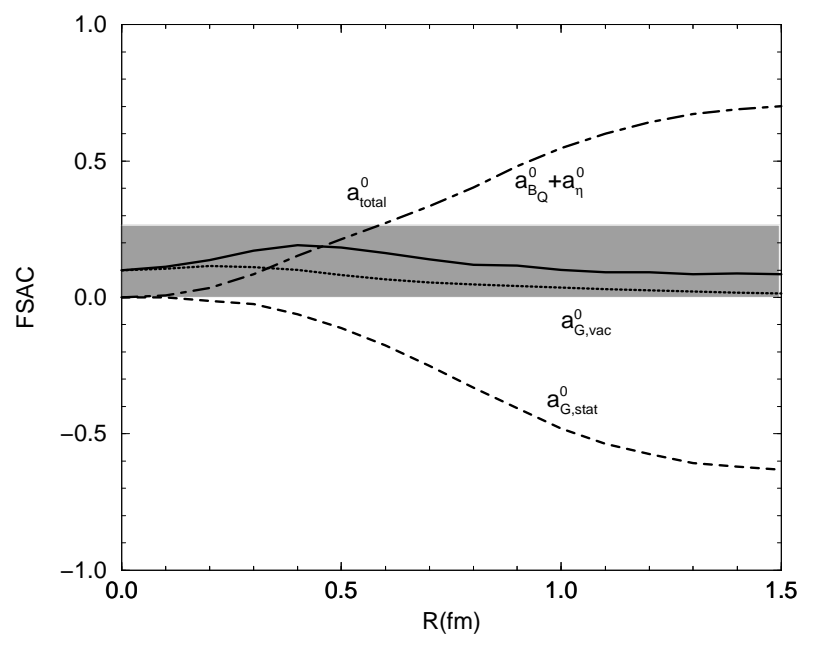

Figure 1: The flavor singlet axial charge of the proton as a function of the bag radius compared with the experiment; it consists of three contributions: (a) matter field contribution: quark plus $\eta\left(a_{B_{Q}}^{0}+a_{\eta}^{0}\right)$, (b) gauge field contribution: the static gluons coupled to the quark source $\left(a_{G, s t a t}^{0}\right)$, (c) Casimir contribution: the gluon vacuum fluctuation $\left(a_{G, v a c}^{0}\right)$, and (d) the sum total $\left(a_{\text {total }}^{0}\right)$. The shaded area represents the range admitted by experiments.

taken into account. It turns out that the interplay between the boundary conditions and Casimir effects plays a crucial role.

Since the flavor-singlet axial current is not conserved because of the anomaly, the color cannot be confined inside the bag unless a suitable boundary condition is put at the surface that cancels the outflow of the color [20]. The boundary term that does this is proportional to the Chern-Simons current on the surface, i.e., the Chern-Simons flux (which is invariant under neither small nor large gauge transformation). This influences the FSAC of the proton nontrivially. Briefly, what happens is that the FSAC contributed by the matter fields (quarks inside the bag and $\eta^{\prime}$ outside the bag) and the FSAC contributed by the gauge field (gluons inside the bag) more or less (or possibly exactly if treated rigorously) cancel, leaving behind only the small contribution from the (gauge field) vacuum fluctuation which is effectively a Casimir effect caused by the boundary with its color-anomalous boundary condition. The cancellation and the remnant small FSAC are shown in Fig. 1.

The upshot of the small result left over which is independent of the size $R$ provides yet another compelling evidence that the proton could be equivalently understood both in terms of quarks/gluons and in terms of macroscopic hadronic variables. When $R$ is taken to be big, it is the QCD variables that take over, e.g., the MIT bag. When the size $R$ is shrunk to a point, the proton is a skyrmion. Thus we have the equivalence of the skyrmionic proton and the quark-gluonic (QCD) proton, that is, the Cheshire Cat. Since there is no way one can exactly bosonize four-dimensional QCD, the equivalence cannot be made exact. We 
simply have an approximate equivalence which can be made more precise by doing more work. What determines the language to use is the kinematic condition of the process one looks at.

\section{Dense Matter and The Cheshire Cat}

The last topic I would like to discuss is infinite nuclear matter at large density. The old lore that at an asymptotic density, the matter can be described by perturbative QCD with weakly interacting boring quarks is simply wrong. What may be happening at high density is something a lot more exciting than that. This explains why lots of people are presently writing papers about it.

What is most surprising and in some sense unexpected is that at high density the Cheshire Cat picture becomes more prominent. In fact, at high density, there ceases to be any real distinction between quarks and hadrons. This can be best seen in terms of a quark soliton analogous to the qualiton Kaplan 222 introduced as a model for the constituent quark. The mechanism I will discuss exploits that at high density diquarks condense giving rise to color superconductivity as recently discussed in the literature [23]. Since the resulting qualiton is formed from a color superconducting ground state, it seems proper to call it superqualiton [25]. It has been argued on general symmetry and dynamical grounds [24] that at high density, hadronic matter of flavor $S U(3)$ is characterized by the condensate

$$
\left\langle q_{L \alpha}^{i a} q_{L \beta}^{j b}\right\rangle=-\left\langle q_{R \alpha}^{i a} q_{R \beta}^{j b}\right\rangle=\kappa \epsilon^{i j} \epsilon^{a b I} \epsilon_{\alpha \beta I}
$$

where $\kappa$ is some constant, $i, j$ are $S L(2, C)$ indices, $a, b$ are color indices, and $\alpha, \beta$ are flavor indices. Equation (4) holds for parity-even states. Such a condensate locks color and flavor so that global color and chiral symmetry are broken to the diagonal subgroup $S U(3)_{C+L+R}$. The consequence of this is that there is an invariant $U(1)$ subgroup that contains a "twisted" photon, measured with which all excitations carry integer charges reminiscent of the HanNambu quarks and have quantum numbers that correspond to those of the mesons and baryons present at zero density. There is then a continuity between the excitations at high density in terms of quarks and gluons and hadronic excitations at low density in terms of baryons and mesons. This clearly is a case of Cheshire Cat.

Now to see that this is the Cheshire Cat in the sense formulated in terms of the chiral bag [18], consider the excitation of a quark on top of the diquark-condensed "vacuum." In [24], such a quark is argued to behave like a baryon. Now I claim that this quark is a quark soliton, i.e., superqualiton 25].

To describe the low-energy dynamics of the color-flavor locking phase, introduce a field $U_{L}(x)$ which maps space-time to the coset space, $M_{L}=S U(3)_{c} \times S U(3)_{L} / S U(3)_{c+L}$. One can take it to be

$$
U_{L a \alpha}(x) \equiv \lim _{y \rightarrow x} \frac{|x-y|^{\gamma_{m}}}{\kappa} \epsilon^{i j} \epsilon_{a b c} \epsilon_{\alpha \beta \gamma} q_{L i}^{b \beta}\left(-\vec{v}_{F}, x\right) q_{L j}^{c \gamma}\left(\vec{v}_{F}, y\right),
$$


where $\gamma_{m}$ is the anomalous dimension of the diquark field of order $\alpha_{s}$ and $q\left(\vec{v}_{F}, x\right)$ denotes the quark field with momentum close to a Fermi momentum $\mu \vec{v}_{F}$. The pairing involves quarks near the opposite edge of the Fermi surface. Similarly, we introduce a right-handed field $U_{R}(x)$, also a map from space-time to $M_{R}=S U(3)_{c} \times S U(3)_{R} / S U(3)_{c+R}$, to describe the excitations of the right-handed diquark condensate. If this field takes a vacuum expectation value as a consequence of the diquark condensation which will, owing to (4), have the form

$$
\left\langle U_{L a \alpha}\right\rangle=-\left\langle U_{R a \alpha}\right\rangle=\kappa \delta_{a \alpha},
$$

then 16 Nambu-Goldstone bosons will get excited \#3. Eight of them will get eaten up by the gluons to give masses to the gluons. The massive gluons then turn into massive vector mesons whose quantum numbers are those of the light-quark vector mesons present at zero density. The remaining eight (pseudoscalar) Nambu-Goldstone bosons are the equivalents of the ones present at zero density and are represented by the interpolating field (5). In analogy to the usual skyrmion at zero density, this field supports a soliton which is a fermion, the quantum numbers of which are identical to those of the usual baryon.

The effective Lagrangian that gives rise to this soliton should in principle be derived from QCD with the help of renormalization group flows toward the Fermi surface of high quark density. Such a derivation would determine the parameters that figure in the effective Lagrangian such as the "pion decay constant" $F$ etc which would carry information on the superconductivity gap etc. At the moment such an effective Lagrangian is not known, so a detailed study of it excitation structure cannot be discussed.

However one can venture to make a few interesting conjectures. Viewed as a superqualiton whose mass is given by the soliton mass, there is nothing that requires that the soliton mass be equal to or near the superconductivity gap $\Delta$ (which is dictated by the condensate). In fact there is nothing which would prevent the mass from being much less than the gap. Thus one could imagine that light fermions are excited within the gap. Correlations between light superqualitons could rearrange the ground state into a different form from that of the standard superconductivity. For this reason the phenomenon of color superconductivity in QCD at high density could be completely different from the usual BCS superconductivity. A similar point in a different context was raised in [26].

\section{Conclusion}

The most important outcome of the recent development of EFT in nuclear physics is that the highly successful approach to nuclear structure using realistic nuclear potentials $(\mathrm{PM})$ is rendered a first-principle interpretation in that it represents the leading term in the EFT expansion with the corrections thereof systematically calculable. This confers the

\footnotetext{
\#3 Actually there are 17 of them, one of which has to do with spontaneous breaking of the baryon number.
} 
power of modern field theory techniques to the standard nuclear physics approach that has been practiced with success since a long time. I am suggesting that this "bridging" comes about thanks to a possible duality between QCD variables and macroscopic (color-singlet) variables that I refer to as the Cheshire Cat Principle. The "proton spin problem" is an illustration of this in the basic structure of the hadron.

In the case of high density, the picture becomes even more intriguing. There we see emerging the symbolic (approximate) equality

$$
\text { "Quark" ₹ "Qualiton" ₹ "Baryon". }
$$

It is amusing that the notion of the Cheshire Cat which was conceived by the need to reconcile the traditional meson-exchange description with the modern QCD description for nuclear processes [27] (i.e., the "little bag" with pion cloud, chiral bag etc) at low density reemerges at high density where one would have expected the bona-fide QCD to be uniquely applicable.

\section{Acknowledgments}

I would like to thank the organizers of this meeting, particularly Chueng-Ryong Ji and Dong-Pil Min for inviting me to give this talk. I would also like to acknowledge the hospitality of KIAS and its Director, C.W. Kim while this paper was being written.

\section{References}

[1] S. Weinberg, Phys. Lett. B 251, 288 (1990) ; Nucl. Phys. B 363, 3 (1991) ; Phys. Lett. B 295, 114 (1992)

[2] M. Rho, Phys. Rev. Lett. 66, 1275 (1991) ; T.-S. Park, D.-P. Min and M. Rho, Physics Reports 233, 341 (1993) ; Phys. Rev. Lett. 74, 4153 (1995) ; Nucl. Phys. A 596, 515 (1996)

[3] C. Ordonez and U. van Kolck, Phys. Lett. B 291, 459 (1992) ; C. Ordonez, L. Ray and U. van Kolck, Phys. Rev. Lett. 72, 1982 (1994) ; Phys. Rev. C 53, 2086 (1996) ; U. van Kolck, Phys. Rev. C 49, 2932 (1994)

[4] D.B. Kaplan, M. Savage and M. Wise, Nucl. Phys. B 478, 629 (1995) ; Phys. Lett. B 424, 390 (1998) ; nucl-th/9801034; nucl-th/9802075; nucl-th/9804032

[5] T.-S. Park, K. Kubodera, D.-P. Min and M. Rho, Phys. Rev. C 58, R637 (1998) ; Nucl. Phys. A 646, 83 (1999)

[6] 1998 and 1999 Joint Caltech-INT Workshops: Nuclear Physics with Effective Field Theory. 
[7] M. Rho, "Chiral symmetry in nuclear physics," nucl-th/9812012.

[8] G.E. Brown and M. Rho, Phys. Rev. Lett. 66, 2720 (1991) .

[9] G.P. Lepage, "How to renormalize the Schrödinger equation?", nucl-th/9706029

[10] D.R. Phillips and T.D. Cohen, "Deuteron electromagnetic properties and the viability of effective field theory methods un the two-nucleon system," nucl-th/9906091.

[11] C.H. Hyun, D.-P. Min and T.-S. Park, "Next-to-leading order np scattering in cut-off effective field theory," to appear.

[12] T.-S. Park, K. Kubodera, D.-P. Min and M. Rho, Astrophys. J. 507, 443 (1998).

[13] T.-S. Park, K. Kubodera, D.-P. Min and M. Rho, nucl-th/9904053, nucl-th/9906005.

[14] J.-W. Chen, G. Rupak and M.J. Savage, "Suppressed amplitudes in $n p \rightarrow d+\gamma$," nucl-th/9905002.

[15] T.M. Müller, private communication; T.M. Müller, D. Dubbers, P. Hautle and O. Zimmer, "Measurement of the $\gamma$ anisotropy in the $\vec{p} \vec{n}, \gamma d$-process," in Proceedings of the "International Workshop on Particle Physics with Slow Neutrons," ILL, Grenoble, France, 22-24 October 1998.

[16] R. Seki and H. Griesshammer, "Open discussion and new ideas," in the Proceedings of the 1999 Joint INT-Caltech EFT Worskshop.

[17] See e.g. B. Frois and J.F. Mathiot, Comments on Nucl. Part. Phys. 18, 307 (1989).

[18] M.A. Nowak, M. Rho and I. Zahed, Chiral Nuclear Dynamics (World Scientific, Singapore, 1996)

[19] A. Hosaka and H. Toki, Phys. Repts. 277, 65 (1996).

[20] H.B. Nielsen, M. Rho, A. Wirzba and I. Zahed, Phys. Lett. B269, 389 (1991); Phys. Lett. 281, 345 (1992).

[21] H.-J. Lee, D.-P. Min, B.-Y. Park, M. Rho and V. Vento, "The proton spin in the chiral bag model: Casimir contribution and Cheshire Cat Principle," Nucl. Phys. A, in press, hep-ph/9810539

[22] D.A. Kaplan, Phys. Lett. B235, 163 (1990); Nucl. Phys. B351, 137 (1991).

[23] M. Alford, K. Rajagopal and F. Wilczek, Phys. Lett. B 422, 247 (1998); R. Rapp, T. Schäfer, E.V. Shuryak and M. Velkovsky, Phys. Rev. Lett. 81, 53 (1998); J. Berges and K. Rajagopal, Nucl. Phys. B 538, 215 (1999); N. Evans, S. Hsu, and M. Schwetz, Nucl.Phys. B551, 275 (1999); Phys. Lett. B 449, 281 (1999); K. Langfeld and M. Rho, 
hep-ph/9811227; G. W. Carter and D. Diakonov, Phys. Rev. D 60, 016004 (1999); M. Alford, J. Berges, and K. Rajagopal, hep-ph/9903502; T. Schwarz, S. Klevansky and G. Papp, hep-ph/9903048; D. T. Son, hep-ph/9812287, Phys. Rev. D 59, 094019 (1999); R. D. Pisarski and D. H. Rischke, nucl-th/9811104; nucl-th/9903023; nucl-th/9906050; D. K. Hong, hep-ph/9812510; hep-ph/9905523; D. K. Hong, I. A. Shovkovy, V. A. Miransky, and L. C. R. Wijewardhana, hep-ph/9906478; T. Schäfer and F. Wilczek, hep-ph/9906512.

[24] T. Schäfer and F. Wilczek, Phys. Rev. Lett. 82, 3956 (1999), hep-ph/9811473; hep-ph/9903503.

[25] D.K. Hong, M. Rho and I. Zahed, "Qualitons at High Density," hep-ph/9906551.

[26] R.D. Pisarski and D.H. Rischke, "Why color-flavor locking is just like chiral symmetry breaking?," nucl-th/9907094.

[27] G.E. Brown and M. Rho, Phys. Lett. B82, 177 (1979). 